\title{
HAPTIC FORCE CONTROL BASED ON IMPEDANCE/ADMITTANCE CONTROL
}

\author{
K. Wen ${ }^{*}$, D. Necsulescu ${ }^{*}$, J. Sasiadek ${ }^{* *}$ \\ *Department of Mechanical Engineering, \\ University of Ottawa, \\ Ottawa, Ontario, Canada, \\ e-mail: necsules@eng.uottawa.ca \\ **Department of Mechanical \& Aerospace Engineering \\ Carleton University \\ 1125 Colonel By Drive \\ Ottawa, Ontario, K1S 5B6, Canada \\ e-mail:jsas@ccs.carleton.ca
}

\begin{abstract}
There are two popular control methods widely used in haptic controller design. First, is impedance control - the operator motion input is measured, and then, the reaction force is fed back to the operator. The alternative method is admittance control forces exerted by operator are measured and positions are fed back to the operator. Both, impedance and admittance control are also two basic methods for interacting with a virtual environment. In this paper, several experiments were performed to evaluate the suitability of impedance control with force feedback for haptic interface development. The difference between conventional application of impedance control in robot motion control and its application in haptic interface development is investigated. Open loop impedance control methodology is implemented for static case and a general-purpose robot under open loop impedance control was developed as a haptic device, while a closed loop model based impedance control was used for haptic controller design in both static and dynamic case. The factors that could affect to the performance of a haptic interface are also investigated experimentally using parametric studies. The experiments were carried out using an experimental setup. Experimental results for 1 DOF rotational motion and 2 DOF planar translational motion systems are presented. Other experimental results are also shown and discussed. Copyright $@ 2005$ IFAC
\end{abstract}

Keywords-- Robotics, Force Control, Impedance and Admittance Control

\section{INTRODUCTION}

Haptic interfaces are one of the technologies that provide the link between a human operator and a remote environment, which is difficult, even not possible for human being to access, or a virtual environment, which is, for some reason, used to emulate a real environment. In the case of virtual 
environment simulation, haptic interface refers to an electro-mechanical system that allows human operators to interact with a virtual environment (simulated with software) through the transmission of interface force/torque and motion.

This system includes a haptic device, mechanical parts, such as a robot arm, electrical parts, such as actuators, sensors and signal conditioning circuits, computer part, including software for the virtual environment simulation and control part, such as the control algorithm used for designing the haptic controller. In fact, a haptic interface is a typical mechatronics system. There are two fundamental and dominant control methods widely used in haptic interface design. First, is to measure the input exerted by human operators and the second is to output the feedback to human operators according to the physical characteristics of virtual reality that it is simulating and let the human operators feel like they are immerged into the virtual environment. Therefore, the two control methods are categorized by different inputs and outputs. When motion input by the user is measured and force is fed back to the user, impedance control is applied. The alternative in (Clover 1997, 1999, Carignan, 2000, 2003, Hogan, 1985, 1987, 1989, and Kazerooni, 1986, 1987, 1990, 1994). Two basic forms of impedances were pointed out. The lower order impedance is the relationship between output force and the input position, i.e. equivalent to the stiffness. In general the impedance is the relationship between the Laplace transforms of force and velocity. Hence, the target dynamics, which include a mass, can be described by a second order mass-damper-spring model and in Laplace domain has the form of $\mathrm{F}(\mathrm{s})=\mathrm{Z}(\mathrm{s}) \mathrm{X}(\mathrm{s})=$ $\left(\mathrm{Ms}^{2}+\mathrm{Cs}+\mathrm{K}\right) \mathrm{X}(\mathrm{s})$. Based on the above analysis, it can be seen that the haptic interface receives the input from a human operator, and then, generates the output according to the physical properties of the virtual environment that is simulated. Impedance control model with feedback was considered in this paper. The impedance control for haptic interface transfer reflects the physical properties of virtual environment to human operator in a different way from the admittance control. Some of the nonlinear aspects of this problem were described in (Kazerooni, 1986, 1987, 1990, 1994).

In haptic interface based on impedance control, the virtual object, in this case the end-effector of robot's arm, is at the beginning in an equilibrium point $\mathrm{X}_{0}$. In the absence of contact, i.e., when the human operator is not interacting with the virtual environment this is a valid assumption. When human operator starts to be in contact with the virtual environment, an interaction force is generated and a deviation from the $\mathrm{X}_{0}$ is also generated as the result, which has the form of impedance and depends on the physical properties of the virtual environment. In this case, the motion deviation from $\mathrm{X}_{0}$ is sensed, and force is fed back to human operator. This process is the first task method, in which forces exerted by the user are measured and positions are fed back to the operator, is call admittance control. Two cases; giving motion input - receiving force feedback, and giving force input- receiving motion feedback are discussed in this paper. In general, for haptic controller design, impedance control is preferred over admittance control due to impedance matching issues. Both, impedance and admittance control were proposed for haptic interfacing by many authors but impedance control was more often used. This can be also explained by the fact that force/torque sensors are more expensive than position sensors. However, in recent research work, force/torque sensors were also used in impedance control for force sensing and feedback. This type of controller is also called impedance controller with force feedback.

\section{IMPEDIANCE AND ADMITTANCE FORCE CONTROL}

Impedance control was extensively analyzed

of the haptic controller. In real world, there are always some physical limits for mechanical systems. Interesting applications were presented in (Hannaford et al, 2002, Volpe, 1990, Jassemi-Zargani, R., and Necsulescu, D.S., 1998). The effects of robot inertial, joint friction, and Coriolis/centripetal forces can be ignored in the static case, but in the dynamic case these effects may be significant and can conceal the physical properties of the virtual environment. An ideal haptic interface design should transfer to the human operator the exact physical properties of the environment and not combine with the effect of the robot dynamics. Consequently, the second task of the haptic controller is to compensate for the mechanical limitations of the haptic device. Admittance control is in fact the reversed form of the impedance control. When a robot is interacting with its environment it must act as impedance while the environment acts as admittance. However, in a haptic system, there are two systems interacting: a human manipulator and a mechanical manipulator. In admittance model, when the human operator starts to be in contact with the virtual environment, the contact force is sensed, and for the given physical properties of the virtual environment this force will result in motion deviation from the case of the absence of the applied contact force. In this situation, the contact force is an input and deviation of motion is an output. Their relationship depends on the physical properties of virtual environment. Haptic controller has also two major tasks: to transfer the physical properties to the human operator and simultaneously, to compensate for the dynamic effects of the haptic device. The impedance control based haptic interface and the virtual environment are shown in Fig.1, based on the following equations: 


$$
X=K I N(\theta)
$$

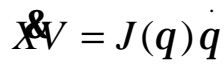

$X^{\ddot{(c)}}=A^{(c)}=M^{-1} B\left(\dot{X}_{0}-\dot{X}\right)+$

$M^{-1} K\left(X_{0}-X\right)-M^{-1} F_{h}$

$\theta^{\ddot{(c)}}=\alpha^{(c)}=J^{-1}(\theta)\left[\ddot{X^{(c)}}-J \dot{(\theta)} \dot{\theta}\right]$

$$
T^{(c)}=I(\theta) \ddot{\theta}+N(\dot{\theta}, \dot{\theta})-J^{T}(\theta) F_{h}
$$

where:

$\theta$ is the $\mathrm{n} \times 1$ vector of angular displacements in joints for n-DOF robot

$\mathrm{X}$ is the $\mathrm{n} x 1$ vector of planar operational space position of the end-effector for $\mathrm{n}-\mathrm{DOF}$ robot

$\mathrm{J}$ is the Jacobian

$\mathrm{X}_{0}$ is the equilibrium operational space position of the end-effector

$F_{h}$ is the internal force in the point of contact of the operator with the end-effector

$\mathrm{T}$ is the torque output of the actuators

I is the moment of inertia function of $\theta$ vector

$\mathrm{N}$ is the viscous friction and Coriolis components

Superscript (c) indicates a commanded value.

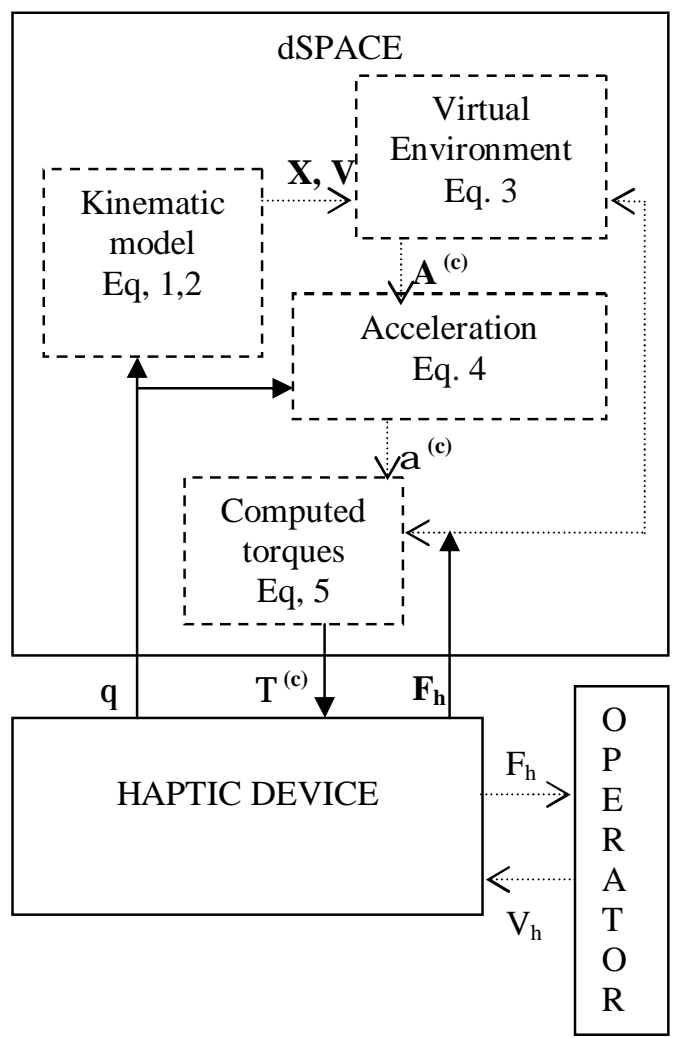

Fig. 1 Block diagram of haptic interface and VE under impedance control

\section{EXPERIMENTS}

The experimental set-up is shown in Fig. 2, where: a) various dSPACE boards are identified:

DS1002 for the communication with a Host PC for program downloading and for data transmission

DS 2001 for acquiring analog signal

DS2101 for providing analog signals outputs

DS3001 for high resolution for acquiring resolver signals.

b) two link planar manipulator with direct drive motors

c) $2 \mathrm{D}$ force sensor at the end-effector

d) drivers for the two direct drive motors

The following experiments were performed:

Experiment 1: Effects of changes in virtual environment parameters versus performance.

The virtual environment in the linear case is described in complex domain by

$\mathrm{F}(\mathrm{s})=\mathrm{Z}(\mathrm{s}) \mathrm{X}(\mathrm{s})\left(\mathrm{Ms}^{2}+\mathrm{Cs}+\mathrm{K}\right) \mathrm{X}(\mathrm{s})$

It is reasonable to assume that the changes of $\mathrm{M}, \mathrm{K}$, $B$ values may have different contributions to the performance of the haptic interface. Further, in critically damped case, this gives better results than other parameters combinations. Experimental parameters and conditions were set as follows:

- sampling rate $1 \mathrm{k} \mathrm{S} / \mathrm{s}$

- cut-off frequency $20 \mathrm{HZ}$ for Butterworth filter used as an anti-aliasing filter

- K=200 N/m

$-\mathrm{M}=4 \mathrm{~kg}, 2 \mathrm{~kg}$

- value of B adjusted to achieve various cases from underdamped to critically damped system.

The performance was judged based on the error between the computed value and the measured actual force. 


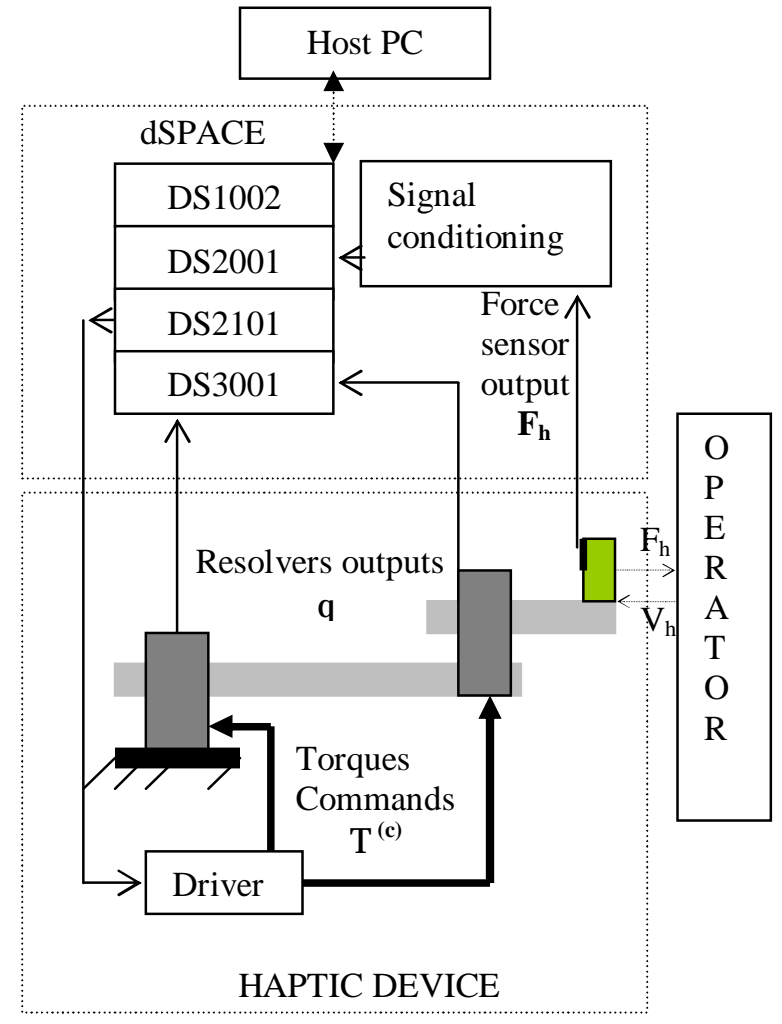

Fig 2. Experimental setup

"Haptic Device" referes to the actual haptic device equipment.

Fig 3 shows experimental results for the second link joint motion due to the motion applied by an operator on the end-effector (q2, vtheta 2 and accq2) and the $\mathrm{y}$-component of the end-effector actual and calculated command force reaction to the operator moves, for $\mathrm{M}=2$ and $\mathrm{B}=30$.

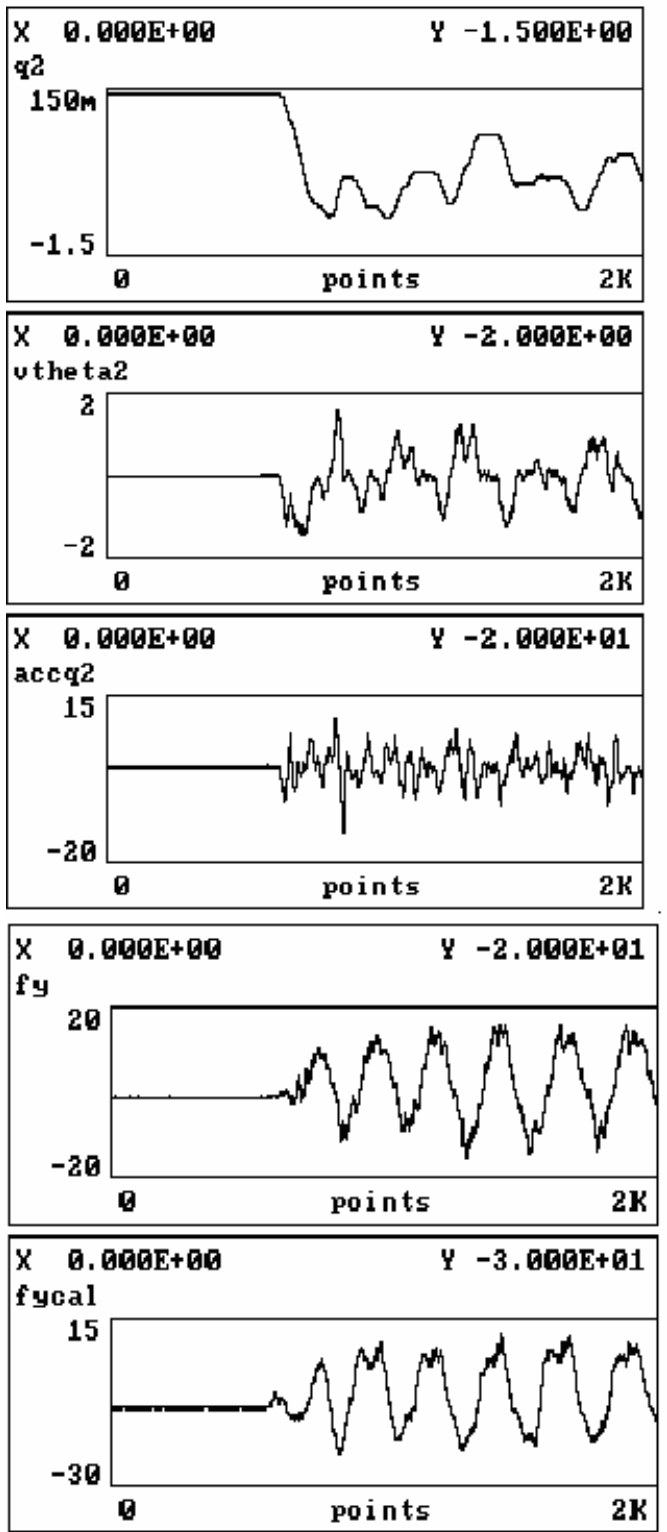

Fig. 3 Experimental results for the second link for $\mathrm{M}=2$ and $\mathrm{B}=30$.

The results show that actual and calculated forces are similar, confirming the satisfactory performance of the impedance control based haptic interface. Experimental results with a variation of the viscosity constant B in the range from 16 to 60 for two values of $\mathrm{M}$ (2 and $4 \mathrm{~kg}$ ), are summarized in Fig. 4. These results show the significant effect of parameters choice on the impedance controller performance.

The error shown in Fig. 4 is between actual and calculated (computed commands) values. 


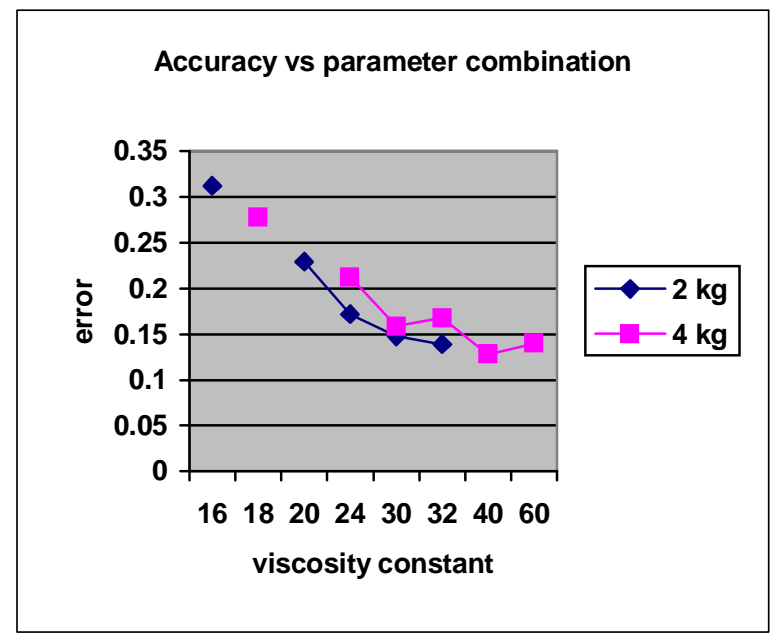

Fig. 4 Error versus change of B

Experiment 2: Effects of changes in sampling rate versus performance.

This experiment was designed to investigate the influence of sampling rate on the performance of haptic interface. Fig. 5 illustrates the experimental results for the second link joint motion due to the motion input and the y-component of the endeffector actual and calculated command force reaction to the operator command. The sampling rate in this test was $1000 \mathrm{~S} / \mathrm{s}$ (Samples per second). Fig. 6 demonstrates the effect of changing the sampling rate to 200, 500 and 800 Samples/sec, respectively, on the error between actual and calculated command force. The cut-off frequency of $20 \mathrm{~Hz}$ requires different coefficients in the equation for different sampling rates. The results show that the error significantly decreases, as the sampling rate is increased.
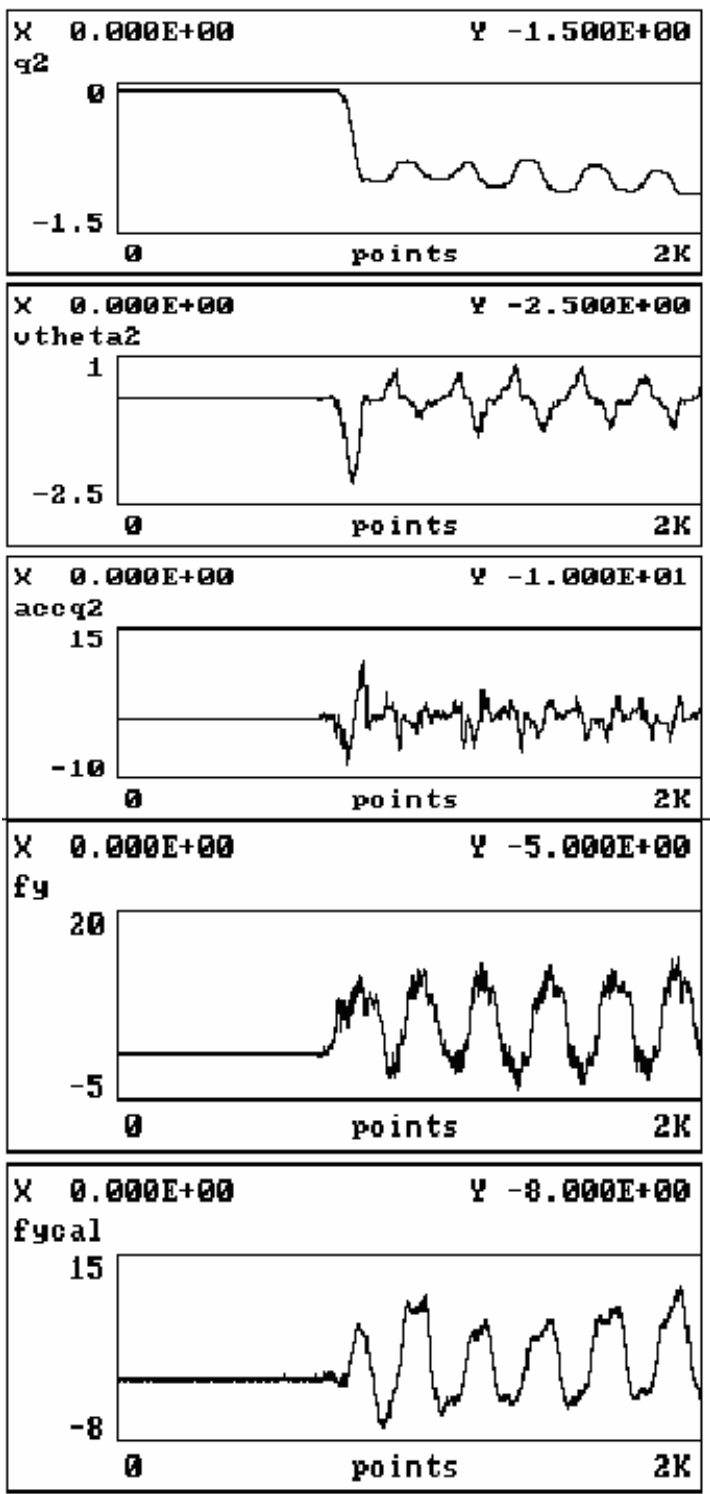

Fig. 5 Experimental results for the second link for the sampling rate of $1000 \mathrm{~S} / \mathrm{s}$.

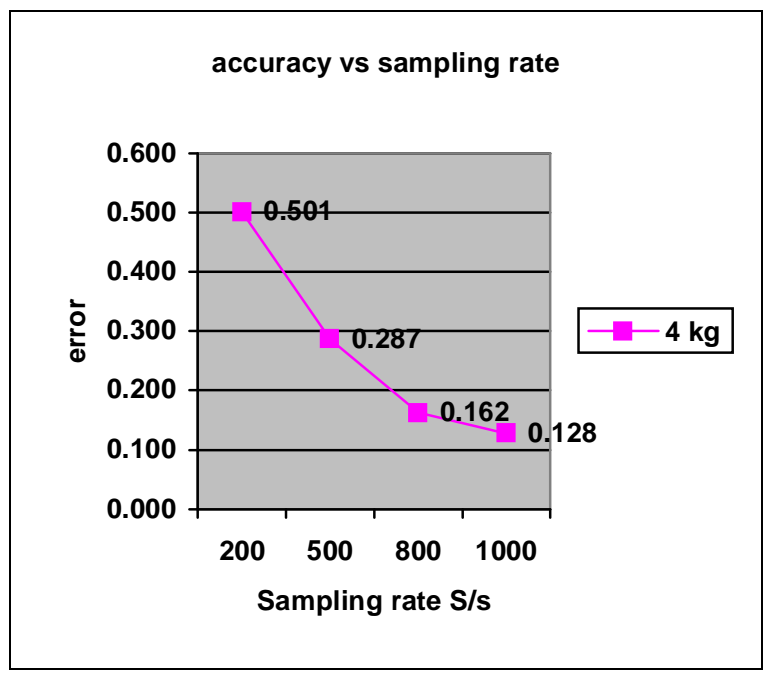

Fig. 6 Error versus change in sample rate 


\section{CONCLUSIONS}

The following conclusions can be drawn from the experiments performed:

1. The difference between conventional applications of impedance control for robot motion control and its application in developing haptic interfaces was analyzed. In haptic interfaces, the dynamic properties of virtual environment are transfered to human operator through a haptic device in the form of mechanical impedance.

2. For the open loop impedance control methodology, implemented for simulation of static case, the errors between measured forces and theoretical values were about $10 \%$. This confirms that, a general-purpose robot under open loop impedance control, can be build as a haptic device for static case.

3. A model based impedance control methodology, introduced and implemented using the experimental setup, proved to be a feasible approach for building haptic interface using a general-purpose robot arm for both dynamic and static case.

4. For a haptic interface, noise, sampling rate and virtual environment parameters could have significant effects on the performance of the haptic interface. These effects, experimentally tested, discussed, and analyzed in this paper show important influence of physical parameters on the overall performance of the haptic device.

In future, model based approach could be used for admittance control in which trajectory following simulation of a virtual object can be simulated. A robot arm with more degrees of freedom will have to be used as a haptic device development system. At the same time, a force/torque sensor with more degrees of freedom is also needed.

\section{REFERENCES}

Necsulescu, D.S., (2001), Mechatronics, Prentice Hall, 2001.

Burdea, G.C., (1996), Force and Touch Feedback Virtual Reality, Wiley.

Clover, C.L., (1999), A control-system architecture for robots used to simulate dynamic force and moment interaction between humans and virtual objects, IEEE Trans. Systems, Man and Cybernetics, C, Vol.29, No.4, pp.481-493.

Clover, C.L., Luecke, G.R., Troy, J.J., and McNeely, W.A., (1997), Dynamic simulation of virtual mechanisms with haptic feedback using industrial robotics equipment, Proc. IEEE Conf. Robotics and Automation, Albuquerque, pp.724-730.

Carignan, C.R. and Akin, David, (2003), Using Robots for Astronaut Training, IEEE Control Systems Magazine.
Carignan, C.R. and Cleary, K.R., (2000), Closedloop force control for haptic simulation of virtual environments, Electron. J. Haptics Res., Vol.2, no. 2 .

Hogan, N., (1985) Impedance control: an approach to manipulation (part 1-theory), The Journal of Dynamic Systems, Measurement, and Control Vol.107, pp.1-7.

Hogan, N., (1985) Impedance control: an approach to manipulation (part II-implementation), The Journal of Dynamic Systems, Measurement, and Control, Vol.107, pp.8-16.

Hogan, N., (1985) Impedance control: an approach to manipulation (part III-Applications), The Journal of Dynamic Systems, Measurement, and Control, Vol.107, pp.17-24.

Hogan, N., (1989), Controlling impedance at the man/machine Interface, in Proc. IEEE Int. Conf. Robotics and Automation, Scottsdale, pp.16261631.

Hogan, N., (1987), Stable Execution of Contact Tasks Using Impedance Control, The Proceedings of the IEEE International Conference on Robotics and Automation, pp.1047-1054.

Kazerooni, H., Human, (1990), Robot Interaction via the Transfer of Power and Information Signals Part I: Dynamics and Control Analysis, IEEE Trans. Systems, Man and Cybernetics, Vol.20, No 2.

Kazerooni, H., Sheridan, T.B. and Houpt, P.K., (1986), Robust Compliant Motion for Manipulators, Part I: The Fundamental Concepts of Compliant Motion, IEEE Journal of Robotics And Automation, Vol.RA-2, No.2, pp. 83-92.

Kazerooni, H., Houpt, P.K. and Sheridan, T.B.,(1986), Robust Compliant Motion for Manipulators, Part II: Design Method, Vol.RA-2, IEEE Journal of Robotics and Automation, No.2, pp.93-105.

Kazerooni, H., (1987), Robust, Non-linear Impedance Control for Robot Manipulators, IEEE International Conference, Vol 4, pp.741750.

Kazerooni, H. and Her, Ming-Guo, (1984), The Dynamics and Control of a Haptic Interface Device, IEEE Transactions on Robotics and Automation, Vol.10, No.4, pp. 453-464.

Hannaford, Blake and Ryu, Jee-Hwan, (2002), Time-Domain Passivity Control of Haptic Interfaces, IEEE Transactions on Robotics and Automation,Vol.18, No.1, pp.1-10.

Volpe, R.A., (1990), Real and Artificial Forces in the Control of Manipulators: Theory and Experiments, $\mathrm{PhD}$ thesis, volpe/papers, pp 58. http://robotics.jpl.nasa.gov/people/

Jassemi-Zargani, R., Necsulescu, D., (1998), Real Time Implementation of the Impedance Control of a Robot Arm, Proc. First IFAC Workshop on Space Robotics, Montreal. 